\title{
User Interface Design and Human-Computer Interaction: A Technique Approach Based on Web Applications
}

\author{
Ying $\mathrm{Liu}^{1, \mathrm{a}}$ \\ ${ }^{1}$ Dalian Art College, Liaoning, China \\ a liuying1232143@126.com
}

Keywords: Human-Computer Interaction,Web Application, Hand tracking and Segmentation

\begin{abstract}
With the rapid development of information science and technology, web based application is everywhere. Designing and implementing the system for human-computer interaction is a hot research area. This work is the part of vision based hand gesture recognition system for Natural Human-Computer Interface. Hand tracking and segmentation are the primary steps for any hand gesture recognition system. The aim of this paper is to develop robust and efficient hand segmentation algorithm where three algorithms for hand segmentation using different color spaces with required morphological processing have were utilized. Hand tracking, and found that the most effective segmentation algorithm to deal with challenges such as skin color detection based on visual system, complex background removal and variable illumination conditions. Our proposed interaction approach could combine the human with the web and related computer application, the experimental analysis illustrates the effectiveness of our methodology.
\end{abstract}

\section{Introduction}

Natural Human Computer Interaction (HCI) is the demand of today's world. Survey and Sign language study shows that from various gesture communications modality, the hand gesture is the most easy and natural way of communication. Real-time vision-based hand gesture recognition is considered to be more and more feasible for Human-Computer Interaction with the help of latest advances in the field of computer vision and pattern recognition [1]. There are various applications using Hand Gesture Recognition. Gaussian distribution is used to establish skin color model, used for segmentation purposes YCbCr color space and Fourier descriptors as feature vector. The BP neural network is used to identify and all of these have led to an improved recognition rate. Highlights and shadows segmentation results, however, found that is not perfect. Guan and Zheng [2] introduced a novel approach to pointing gesture recognition based on binocular stereo vision, in which user needs to wear special clothes or markers and was found to be suitable for both left and right handed users. Freeman and Weissman [3] explained television control application by hand gesture. In Real-time Vision based Hand Gesture recognition system, hand tracking and segmentation are most important and challenging steps towards gesture recognition. Uncontrolled environment, lighting condition, skin color detection, rapid hand motion and self-occlusions are the challenges need to be considered while capturing and tracking the hand gesture. Various researchers are working on hand tracking and segmentation to make it robust to achieve natural interface with machine. Bao et al. introduced a new robust algorithm called Tower method for hand tracking module where, skin color was considered for hand gesture tracking and recognition.

Various gray-level segmentation techniques and image representation approaches, such as use of single threshold value, adaptive thresholding, P-tile method, edge pixel method, iterative method and use of fuzzy set are available for object segmentation. Wang[4] proposed a novel kernel based image representation method. Approximation of the hand morphology was accomplished by SGONG (self-growing and self-organized neural gas) Network. Algorithm was able to identify finger, palm center, hand center, hand slope as well as finger features for recognition purpose. Probability based classification method was used for gesture classification. Skin color detection and complex background are major challenges in hand gesture recognition. Binary linked objects are groups of 
pixels that share the same label due to their connectivity in a binary image. Burande et al. implemented Blobs analysis technique for skin color detection under complex background. Kalman filtering, HMM and Graph matching algorithm were used for gesture recognition. Howe et al. had introduced fusion of skin color and motion segmentation. Face and hand of signer were successfully detected by using skin color segmentation. False detection of skin region in the uncontrolled background also occurs due to light variation, so motion segmentation was used to find the difference between the moving foreground object and the stationery background.

\section{Hand Gesture Recognition Model.}

Let $S$ be a hand gesture recognition system that recognizes hand gesture. $S=\{I, G, M, F, O\}$, I is a set of input hand gestures, $G$ represents a set of single-handed anticipated static gestures, $M$ represents mouse operation such as left click, right click, F represents feature vector for $G, O$ represents output with application interface (A). $G=\left\{G_{1}, G_{2}, \mathrm{~L}, G_{7}\right\}$ represents the set of elements.

\section{Color Models.}

The aim of the proposed project is to overcome the challenge of skin color detection for natural interface between user and machine. So to detect the skin color under dynamic background the study of various color models was done for pixel based skin detection. In this paper, three color spaces has been chosen which are commonly used in computer vision applications. RGB: Three primary colors $\operatorname{red}(R)$, green(G), and blue(B) are used. The main advantage of this color space is simplicity. However, it is not perceptually uniform. It does not separate luminance and chrominance, and the R, G, and B components are highly correlated. HSV (Hue, Saturation, Value): It express Hue with dominant color (such as red, green, purple and yellow) of an area. Saturation measures the colorfulness of an area in proportion to its brightness. CIE -Lab: It defined by the International Commission on Illumination. It separates a luminance variable L from two perceptually uniform chromaticity variable(a,b).

\section{Hand Segmentation}

Gesture Set.

Static gesture is a specific posture assigned with meaning. Following are the static gesture set specified for the proposed system with the specific meaning. Application interface will be provided after recognition of specified posture for action. Simplicity and user frendliness were taken into consideration for the design of anticipated posture set. For the mouse cursor movement the center of the hand gesture window was passed as a mouse cursor. Figure 1. shows anticipated static gestures set with defined task.
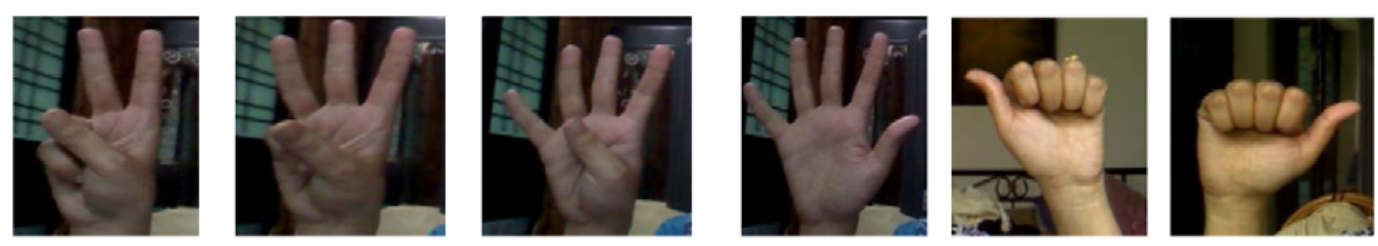

Fig. 1 The Gesture Set

\section{Hand Segmentation Using HSV.}

A Novel Approach for Image segmentation algorithm has been developed and tested for green color glove. In this approach, color based segmentation was attempted using HSV color space. The H, $\mathrm{S}$ and $\mathrm{V}$ separation was done using following equations.

$$
\begin{aligned}
& V=\max \{R, G, B\} \\
& \delta=V-\min \{R, G, B\} \\
& S=\delta / V
\end{aligned}
$$

The input image of green color samples was passed to the algorithm and from H-S histogram the $\mathrm{H} \_$range $=\left[\begin{array}{l}0.40 .550 .60 .6 \\ 0\end{array}\right]$ and S_range $=\left[\begin{array}{l}0.2 \\ \text { 1.0.0 }\end{array}\right]$ were experimented for segmentation. Algorithm could able to subtract dynamic background. Skin color samples needed to be passed to the algorithm 
for skin color detection. The drawback of this algorithm was training samples of the color need to be stored. It was sensitive to little variation in color brightness. The process is: (1) Capture the Image, (2) Read the input image, (3) Convert RGB image into lab color space, (4) Convert the color values in I into color structure specified in cform, (5) Compute the threshold value, (6) Convert Intensity image into binary image, (7) Performing morphological operations such as erosion.

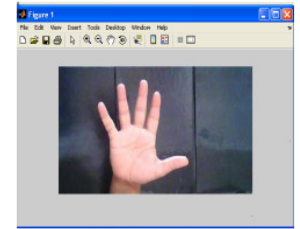

\section{Hand Tracking Algorithm.}

The prime objective of this algorithm was robust skin color detection and removal of complex background. The limitation of previous two techniques was overcome with the hand tracking algorithm. In this approach, hand detection and segmentation were attempted. Hand tracking was done using mean shift algorithm [5]. Odd frame has been considered for fast processing. For robust performance user's skin color sample was passed and HSV histogram was created. Experimented threshold value has been used for the segmentation. Algorithm was found to be working for dynamic color tracking under complex background and able to segment required hand image on which image morphological operations were applied to get the contour. CamShift function (a variation on mean shift algorithm) within the OpenCV library is used for tracking and detection. Edge traversal algorithm was applied for getting fine contour of the hand shape. As dynamic background was considered, while capturing the user's gesture after edge detection, there was a possibility to detect unwanted edges from the background. In an attempt to only identify the boundary of user's hand edge, traversal algorithm was devised [6]. Figure 3 explains the flow chart of HTS algorithm.

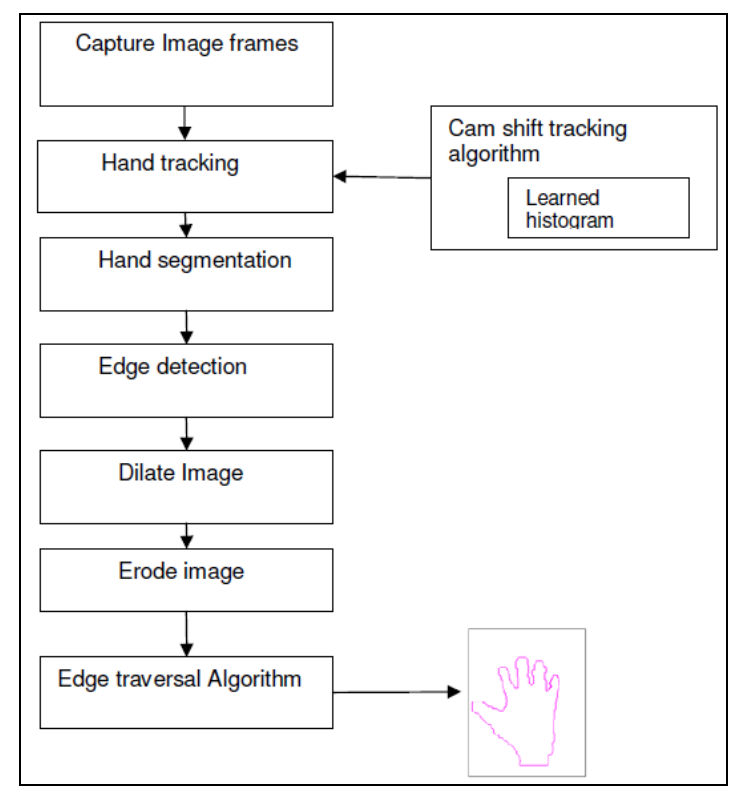

Fig. 3 The HTS Flowchart

\section{Experimental Analysis}

HTS algorithm was tested on four users for five gestures. Instances of three to five gestures on same users were tested. Algorithm was tested on green color glove and skin color on 70 samples. It was observed that using green color glove segmentation process is faster than using skin color due the varying lighting condition. Figure 4 shows segmented results using green color gloves and Figure 5 shows segmented results on skin color. 


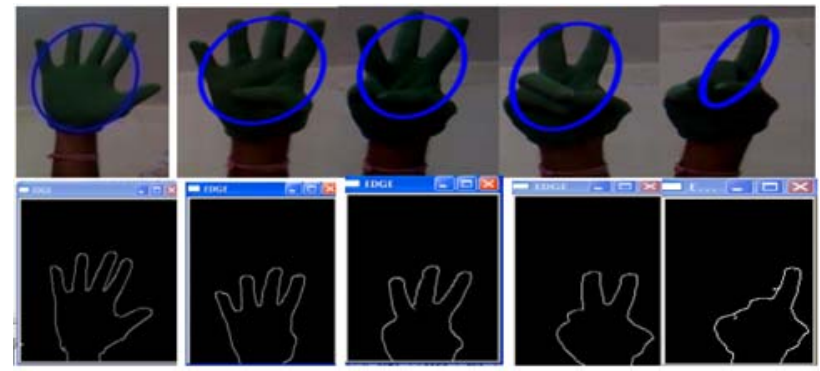

Fig. 4 The Segmentation Results

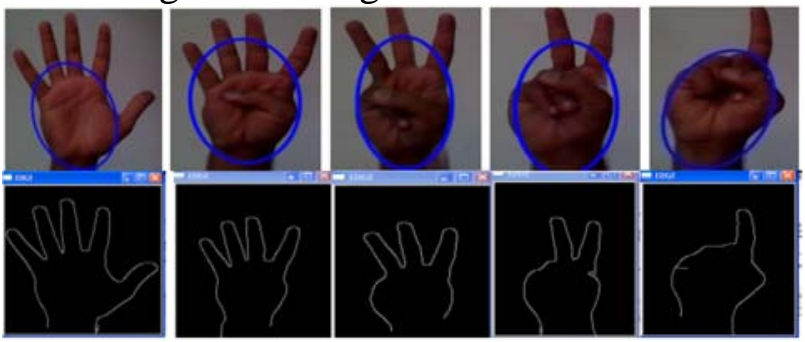

Fig. 5 The Segmentation Results on Skin Color

\section{Summary and Conclusion}

This is an ongoing project and first phase of the project where we tried to implement and tested various techniques for efficient and robust segmentation. Better results on skin color detection using HTS algorithm were achieved, where for fast hand tracking, odd frames for processing were considered. The next phase of the project is creation of multiple feature vectors for desired classification and recognition accuracy. Geometric features, such as line identification using Hough transform , Fourier descriptor, and Image Hu moments will be used due to the properties of rotation, scale and translation invariant. Genetic algorithm will be used for gesture recognition. Window APIs (Application Program Interface) will be used for specified action after Gesture recognition. In the future, we plan to do more user experience research to modify our work.

\section{References}

[1] V. Pavlovis,R. Sharma and T. huang, "Visual Interpretation of Hand Gesture for HumanComputer Interaction: A Review", IEEE Transaction on pattern Analysis and Machine Intelligence, Vol. 19, No.7, Jul 1997.pp.,677-695.

[2] Y. Guan, M. Zheng, "Real-time 3D pointing gesture recognition for natural HCI”, Proceedings of the world congress on Intilligent Control and Automation, China, pp, .2433-2436.,2008.

[3] P. Bao, N. Binh, T. Khoa, “A new Approach To Hand Tracking and Gesture Recognition By A New Feature Type And HMM”, International Conference on Fuzzy Systems and Knowledge Discovery, IEEE Computer Society, 2009.

[4] Wang, Haoxiang, and Jingbin Wang. "An Effective Image Representation Method using Kernel Classification."

[5] C. Burande,R. Tugnayat, N. Choudhary, "Advanced Recognition Techniques for Human Computer Interaction”, IEEE, Vol 2 pp., 480-483,2010.

[6] C.Jung, C.Kim, S.Chae, and S. Oh, "Unsupervised Segmentation of Overlapped Nuclei Using Bayesian Classification”, IEEE Transaction on Biomedical Engineering, Vol. 57, No.12,2010. 